\title{
Viabilidade do tratamento de água residuária sintética têxtil em reator aeróbio de leito fixo
}

\section{Viability of the synthetic textile wastewater treatment in a fixed-bed aerobic reactor}

\author{
Kelly Rodrigues \\ Engenheira Civil pela Universidade Estadual do Maranhão (UEMA). Doutora em Hidráulica e Saneamento pela Escola de Engenharia de São Carlos, \\ Universidade de São Paulo (EESC-USP). Professora efetiva do Departamento de Química e Meio Ambiente do Instituto Federal de Educação Ciência e \\ Tecnologia do Ceará (IFCE)
}

\section{Carla Bastos Vidal}

Tecnóloga em Processos Químicos pelo IFCE. Mestranda do curso de Saneamento Ambiental da Universidade Federal do Ceará (UFC)

\section{Bárbara Chaves Aguiar Barbosa}

Tecnóloga em Gestão Ambiental pelo IFCE

\section{Carlos Ronald Pessoa-Wanderley}

Engenheiro Civil e Mestre em Saneamento Ambiental pela UFC. Professor efetivo do Curso de Engenharia Ambiental do IFCE

\section{Iolanda Cristina Silveira Duarte}

Bióloga pela Universidade Estadual Paulista "Júlio de Mesquita Filho" (Unesp) de Assis. Doutora em Hidráulica e Saneamento pela EESC-USP. Professora do Curso de Biologia da Universidade Federal de São Carlos (UFSCar) de Sorocaba

\section{Glória Marinho}

Farmacêutica pela UFC. Doutora em Hidráulica e Saneamento pela EESC-USP. Professora efetiva do Departamento de Química e Meio Ambiente do IFCE

\section{Resumo}

Um reator de escoamento contínuo, inoculado com Aspergillus niger AN400, possuía volume total de $5 \mathrm{~L}$ e foi operado a $29^{\circ} \mathrm{C}$, com oito horas de tempo de detenção hidráulica e $150 \mathrm{~L} . \mathrm{h}^{-1}$ de vazão de ar, para remover $25 \mathrm{mg} \cdot \mathrm{L}^{-1}$ de corante vermelho do congo de água residuária sintética. A alimentação do reator foi realizada em duas fases: Fase I, com 0,5 g.L-1 de sacarose e Fase II, sem sacarose. Na Fase I, foi possível observar eficiências de remoção de matéria orgânica (mg de DQO.L-1 ) e de cor (mg PT.L-1) de $80 \pm 16 \%$ e $82 \pm 10 \%$ (mg Pt.L-1 ), respectivamente. Na Fase Il, a eficiência de remoção de matéria orgânica foi de $75 \pm$ 13\% e de cor (mg Pt.L-1) de $89 \pm 7 \%$. As maiores remoções de nutrientes foram alcançadas pelo reator na Fase I, com 25\% de amônia, 90\% de nitrito, 93\% de nitrato e $21 \%$ de fósforo. Aparentemente, a presença de sacarose melhorou a remoção dos nutrientes.

Palavras-chave: Aspergillus niger AN400, Escoamento contínuo, Remoção de nutrientes, Vermelho do congo

\begin{abstract}
A continuous flow reactor, inoculated with Aspergillus niger AN400, with total volume of $5 \mathrm{~L}$ was operated at $29^{\circ} \mathrm{C}$, with eight hours of retention hydraulic time and $150 \mathrm{~L} . \mathrm{h}^{-1}$ of air flow rate in order to remove $25 \mathrm{mg} \cdot \mathrm{L}^{-1}$ of Congo Red dye from a synthetic wastewater. The feeding of the reactor, inoculated with Aspergillus niger AN400, was done in two phases: Phase I, with $0,5 \mathrm{~g} / \mathrm{L}$ of saccharose and Phase II, with no saccharose. In Phase I, it was possible to verify efficiencies of organic matter and color (mg Pt. $\mathrm{L}^{-1}$ ) removal of $80 \pm 16 \%$ and $82 \pm 10 \%$, respectively. In Phase II, the efficiency of organic matter removal was $75 \pm 13 \%$ and color removal was $89 \pm 7 \%$. The higher removals of nutrients were achieved by the reactor in Phase I with $25 \%$ to ammonia, $90 \%$ to nitrite, $93 \%$ to nitrate and $21 \%$ to phosphorus. Apparently, the presence of saccharose improved the removal of the nutrients.
\end{abstract}

Keywords: Aspergillus niger AN400, Continuous flow, Nutrients removal, Congo red. 


\section{Introdução}

O parque têxtil é um importante setor da economia brasileira, sendo que o Brasil é um dos dez maiores produtores mundiais de fios, tecidos e malhas, ocupando o terceiro lugar na produção de tecidos de algodão (CAMPOS; DE PAULA, 2006). Em contrapartida, as indústrias têxteis são responsáveis pelo uso intenso de corantes dos mais variados e pela produção de grande volume de efluentes de carga orgânica elevada e presença de corantes, os quais, durante o processo de tingimento, não se aderem à fibra e acabam sendo descartados juntamente com o efluente final, gerando sérios problemas ambientais devido à sua potente ação poluidora tanto no solo quanto na água (GUARATINI; ZANONI, 2000; VAN DER ZEE et al, 2005; YANG et al, 2008).

Os corantes têxteis são compostos químicos de difícil biodegradação e podem causar prejuízos sérios não somente ao ambiente, mas também à saúde humana, pois esses corantes e/ou seus subprodutos, aminas aromáticas, são carcinogênicos e mutagênicos (KHELIFI et al, 2008). Assim, diferentes técnicas e processos vêm sendo utilizados visado à sua remoção dos efluentes industriais, a partir da ruptura da ligação azo, a qual está presente em cerca de 70\% dos corantes utilizados nas indústrias têxteis e constitui o principal obstáculo para sua biodegradação (VAN DER ZEE et al, 2005). Segundo Van Der Zee et al (2005), os processos físico-químicos possuem custo elevado e versatilidade limitada, além de sofrerem interferência de outros constituintes do efluente. Por outro lado, os processos biológicos são menos onerosos e representam uma alternativa viável para a eliminação dos corantes de efluentes têxteis, além de reduzir a carga orgânica desses efluentes (MÉNDEZ-PAZ; OMIL; LEMA, 2005; NACHIYAR; RAJAKUMAR, 2004).

Nos processos biológicos, os micro-organismos são responsáveis pela metabolização e adsorção dos pigmentos responsáveis pela cor. Porém, a biodegradação de corantes azo frequentemente exige a utilização de processos sequenciais anaeróbio/aeróbio, com a ligação azo rompida pelos micro-organismos, sob condições anaeróbias. As aminas aromáticas formadas durante o processo anaeróbio somente são mineralizadas eficientemente em meio aeróbio, quando se pode prever a diminuição da concentração de nutrientes de forma mais consistente (ONG et al, 2008).

Com relação ao tratamento de efluentes têxteis com a utilização de fungos, embora boa parte dos trabalhos encontrados seja com uso de biomassa morta (FU; VIRARAGHAVAN, 2002; KUMARE; ABRAHAM, 2007; ARICA; BAYRAMOGLU, 2007; PATEL; SURESH, 2008), estudos com emprego de biomassa viva, inoculada em reatores aeróbios, vêm trazendo bons resultados, particularmente quanto à remoção de cor e matéria orgânica carbonácea nestas unidades de tratamento, conforme observado nas pesquisas de D'Souza et al (2006) e Santos et al (2007) e Sharma et al (2009). Resultados ainda mais animadores têm sido observados com o uso de reatores com fungos como unidade de tratamento, visando à remoção de corantes têxteis, conforme relatado nos trabalhos de Pessoa-Wanderley (2007), Svobodová et al (2007) e Yang et al (2008), dispensando-se o pós-tratamento dos efluentes destes reatores em face de excelentes remoções de corante e de matéria orgânica carbonácea, indicando ainda produção baixa de aminas aromáticas.

Nesta pesquisa, foi estudado o tratamento biológico de água residuária têxtil sintética em reator inoculado com a espécie fúngica Aspergillus niger AN400, tendo como objetivo a remoção do corante vermelho do congo, de matéria orgânica carbonácea e de nutrientes na presença (Fase I) e ausência de sacarose (Fase II).

\section{Material e Métodos}

\section{Água residuária sintética têxtil}

A água residuária sintética têxtil foi preparada conforme descrito em Araújo (2008), com água de torneira, acrescida de $25 \mathrm{mg} . \mathrm{L}^{-1}$ do azo corante Vermelho do congo (Figura 1), macronutrientes nas seguintes concentrações em mg.L-1: 280 de $\mathrm{NH}_{4} \mathrm{Cl}, 250$ de $\mathrm{K}_{2} \mathrm{HPO}_{4}$, 100 de $\mathrm{MgSO}_{4} \cdot 7 \mathrm{H}_{2} \mathrm{O}$ e 10 de $\mathrm{CaCl}_{2} \cdot 2 \mathrm{H}_{2} \mathrm{O}$. Foram adicionados ainda 0,5 g. $\mathrm{L}^{-1}$ de sacarose, como cossubstrato, e $1 \mathrm{~mL}$ de solução contendo micronutrientes (mg. $\mathrm{L}^{-1}$ ): 50 de $\mathrm{H}_{3} \mathrm{BO}_{3}, 50$ de $\mathrm{ZnCl}_{2}, 2000$ de $\mathrm{FeCl}_{2} \cdot 4 \mathrm{H}_{2} \mathrm{O}, 500$ de $\mathrm{MnCl}_{2} \cdot 4 \mathrm{H}_{2} \mathrm{O}, 38$ de $\mathrm{CuCl}_{2} \cdot 2 \mathrm{H}_{2} \mathrm{O}, 90$ de $\mathrm{AlCl}_{3}$. $\mathrm{H}_{2} \mathrm{O}, 50$ de $\left(\mathrm{NH}_{4}\right) 6 \mathrm{Mo}_{7} \mathrm{O}_{24} 4 \mathrm{H}_{2} \mathrm{O}$ e 2000 de $\mathrm{CoCl}_{2} \cdot 6 \mathrm{H}_{2} \mathrm{O}$. A água residuária foi acidificada com ácido clorídrico PA até pH 4,0 a fim de manter o meio mais propício ao desenvolvimento do Aspergillus niger e minimizar a ação de bactérias.

\section{Obtenção do inóculo fúngico}

O inóculo foi obtido a partir do cultivo e produção da espécie fúngica e de acordo com os procedimentos descritos em Sampaio (2005). A espécie Aspergillus niger AN400 foi cultivada em placas de Petri com meio de cultura Agar Sabouraud Dextrose, acrescido de 1 $\mathrm{mL}$ da solução de Vishniac por litro de meio de cultura. Foi utilizado 0,05 g. $\mathrm{L}^{-1}$ de cloranfenicol a fim de minimizar o crescimento de bactérias.

As placas permaneceram à temperatura de $\pm 28^{\circ} \mathrm{C}$, durante dez dias, após os quais os esporos de Aspergillus niger AN400 foram removidos com auxílio de alça estéril e $4 \mathrm{~mL}$ de solução de Tween 80 , sendo a suspensão de esporos transferida para tubos de ensaio. Para contagem dos esporos, foi preparada uma solução utilizando-se $50 \mu \mathrm{L}$ da suspensão previamente agitada em agitador tipo Vórtex, acrescida de $950 \mu \mathrm{L}$ de solução Tween 80, resultando em diluição de 1:20. Em seguida, foram transferidos $20 \mu \mathrm{L}$ da solução preparada para câmara de Newbauer para realizar a contagem dos esporos em microscópio óptico com aumento de 400 vezes. 


\section{Imobilização dos fungos no meio suporte}

O reator foi preenchido com o meio suporte e, em seguida, alimentado com meio de crescimento adaptado de Rodrigues (2006), acrescido de 0,05 g.L-1 de cloranfenicol. Adicionou-se o inóculo na concentração de $2 \times 10^{4}$ esporos. $\mathrm{L}^{-1}$, mantendo-se o reator sob aeração do seu conteúdo durante 24 horas, com introdução de ar na vazão de $150 \mathrm{~L} . \mathrm{h}^{-1}$. Após este período, houve recirculação de 100\% da vazão efluente ( $7 \mathrm{~L} \mathrm{dia}^{-1}$ ) durante quatro dias, até que foi observada a formação visual do biofilme na superfície do material suporte, quando o sistema começou a ser operado em regime de escoamento contínuo, sem recirculação.

\section{Operação e monitoramento do reator aeróbio de leito fixo}

O reator aeróbio de leito fixo e escoamento ascendente (Figura 2) foi confeccionado em acrílico, com volume total de 5 L, diâmetro interno de $90 \mathrm{~mm}$ e $80 \mathrm{~cm}$ de altura, com dispositivos de entrada de afluente e saída de efluente e, ainda, entrada de ar, cujo fornecimento foi realizado por minicompressor de ar, mantendo-se a mesma vazão de aeração no momento da imobilização da biomassa no material suporte (150 L.h ${ }^{-1}$ ), sendo responsável ainda por manter o conteúdo do meio agitado, funcionando o reator no regime de mistura perfeita, conforme Rodrigues (2006). Foram empregados como meio suporte inerte $235 \mathrm{~g}$ de manta de polietileno, cortada em quadrados de 2 × 2 $\mathrm{cm}$, acomodados dentro do reator em redes de polietileno.

O reator biológico foi operado com tempo de detenção hidráulica (TDH) de oito horas, dividido em duas fases de alimentação, conforme a presença de cossubstrato: com adição de 0,5 g.L.-1 de sacarose (Fase I) e sem adição de sacarose (Fase II), as quais tiveram duração de 54 e 21 dias, respectivamente. A temperatura da sala de operação do reator foi de $\pm 29^{\circ} \mathrm{C}$. A menor duração da operação do reator na Fase II decorreu de problema estrutural no laboratório onde o experimento foi executado, de modo que ficou inviabilizada a continuidade da pesquisa que visava à coleta de maior aporte de dados nesta fase.

\section{Variáveis determinadas}

As variáveis físico-químicas determinadas no presente estudo foram: demanda química de oxigênio (DQO) da amostra bruta e filtrada, cor real - em miligramas de platina por litro (mg Pt. $\left.L^{-1}\right)$ - amônia, nitrito, nitrato e fósforo. As análises foram realizadas com base na metodologia sugerida por APHA (1998).

A determinação da cor também foi realizada a partir da medida da absorção do grupo cromóforo do vermelho do congo no comprimento de onda $(\lambda)$ de $500 \mathrm{~nm}$, de acordo com os procedimentos descritos por Dos Santos (2001) e Silva Filho (2006), tendo se estabelecido ainda a relação entre a referida medida e o seu equivalente em termos de concentração

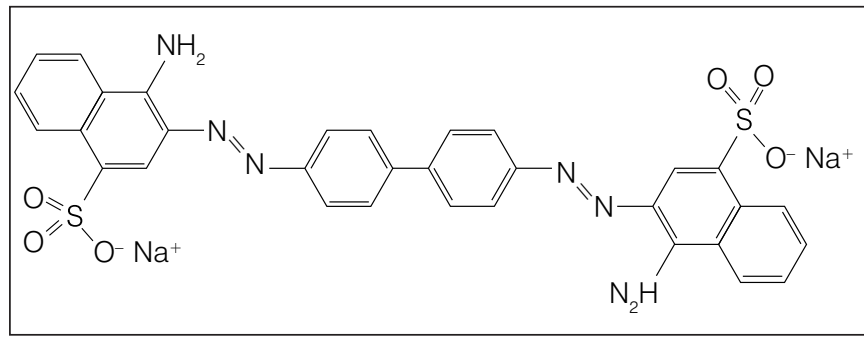

Figura 1 - Estrutura molecular do corante vermelho do congo

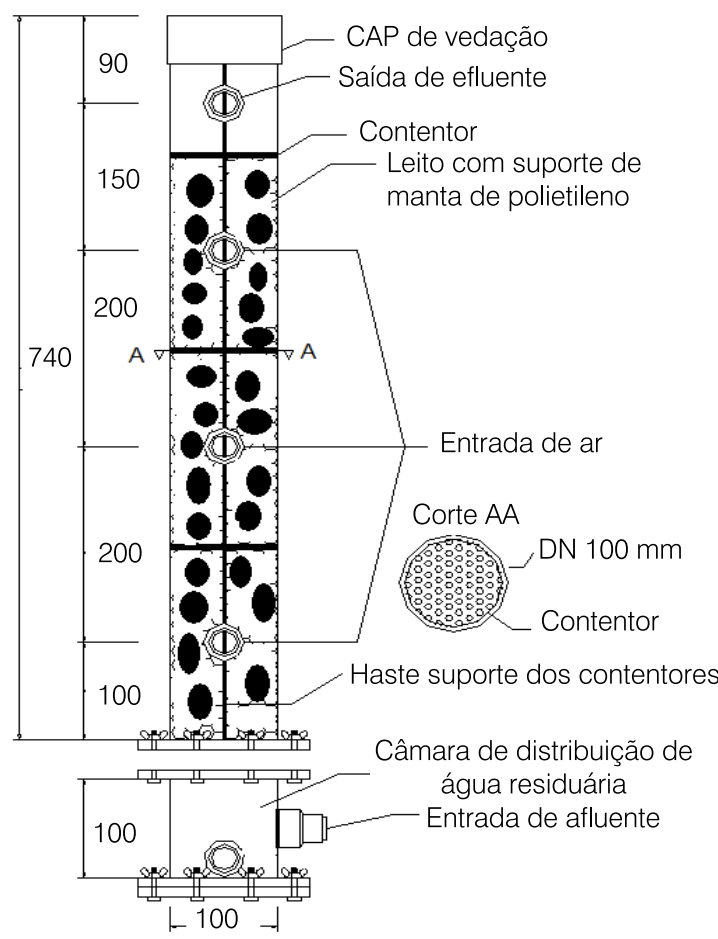

Medidas do desenho em milímetro (mm)

Figura 2 - Detalhes do reator com leito fixo e escoamento ascendente com meio suporte de manta de polietileno e contentores para evitar deslocamento ascensional do material suporte

de corante. Foi construída uma curva de calibração com o uso de soluções de concentrações conhecidas de vermelho do congo, variando de 0 (branco) a 50 mg. $\mathrm{L}^{-1}$, sendo que, antes da leitura, em espectrofotômetro, as amostras foram centrifugadas a $3.500 \mathrm{rpm}$, durante 15 minutos. Desta forma, a partir dos dados de absorbância versus concentração de corante adicionado, pôde ser obtida a equação que estabeleceu a relação entre concentração de corante equivalente e absorbância.

\section{Ensaio de adsorção no material suporte}

O ensaio de adsorção determinou a parcela de corante responsável pela saturação da manta de polipropileno via adsorção, o qual foi realizado de acordo com os procedimentos descritos em Rodrigues (2006). 


\section{Análises microscópicas}

No final da operação do reator, foram retiradas amostras do biofilme, as quais foram submetidas a exames microscópicos. O biofilme aderido à manta suporte foi removido com ajuda de pérolas de vidro e água destilada esterilizada a $121^{\circ} \mathrm{C}$ a $1 \mathrm{~atm}$, procedendo-se posteriormente a diluições de $10^{-1}, 10^{-2}$ e $10^{-3}$ para melhor visualização das estruturas dos micro-organismos no momento do uso do microscópio óptico. Foi retirado $1 \mathrm{~mL}$ de cada diluição para adição em placas de Petri, contendo meio de cultura Saboraud Dextrose, as quais foram mantidas em estufa a $\pm 30^{\circ} \mathrm{C}$ durante uma semana para verificação das colônias. Amostras das placas que receberam as diluições foram fixadas em lâminas com ágar, realizando-se, em seguida, a microscopia em microscópio óptico ACROM L1000, com aumento de até 1.600 vezes.

\section{Resultados e Discussões}

O afluente apresentou, em relação à cor real, $510 \mathrm{mg} \mathrm{Pt.L}^{-1} \pm 37$, sendo que a concentração de corante foi, em média, de $14 \mathrm{mg} \cdot \mathrm{L}^{-1} \pm$ 5 , valor dentro da concentração média registrada em efluentes das indústrias do Parque Industrial de Maracanaú, no Ceará (SILVA FILHO, 2006). A variação da cor real no afluente e efluente do reator é apresentada na Figura 3.

Apesar de se tratar de água residuária sintética, houve oscilação da cor real tanto em mg Pt. $L^{-1}$ quanto em relação ao valor da absorção do grupo cromóforo do vermelho do congo ( $\lambda 500 \mathrm{~nm}$ ). Da mesma forma, quase sempre, a concentração do corante no afluente ficou abaixo do proposto (25 mg. $\left.\mathrm{L}^{-1}\right)$ ao longo da operação do reator, fato atribuído à adsorção do corante nas paredes do reservatório de armazenamento da água residuária.

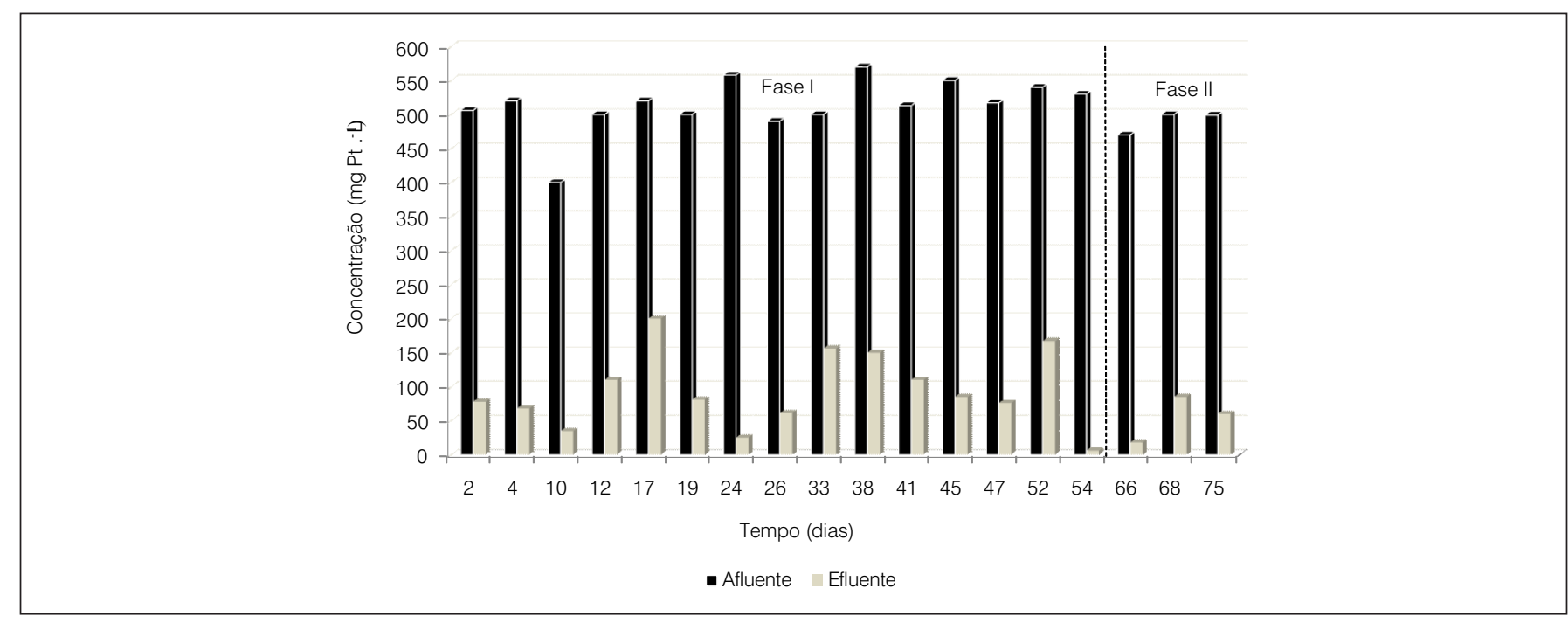

Figura 3 - Variação da cor real no afluente e efluente do reator biológico inoculado com Aspergillus niger AN400

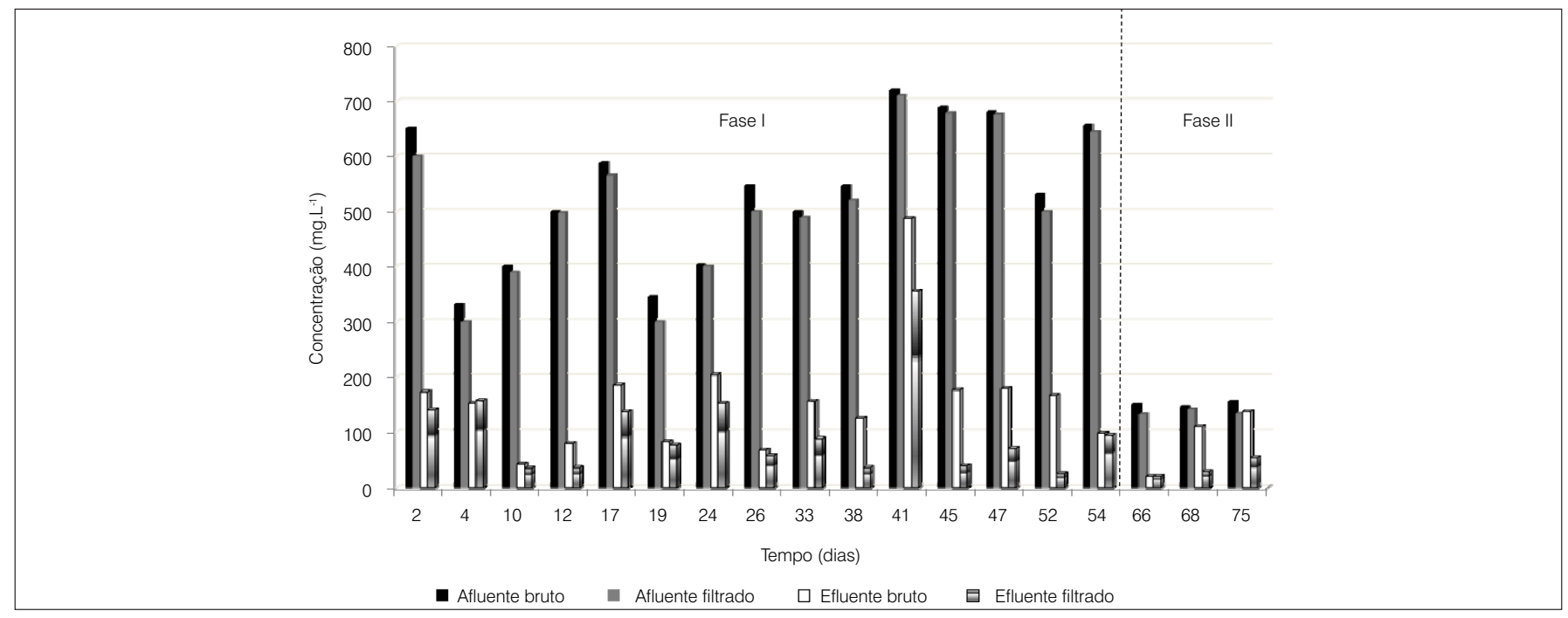

Figura 4 - Variação da concentração de matéria orgânica, medida em DQ0, amostra bruta e filtrada no afluente e efluente do reator biológico inoculado com fungos 
$\mathrm{Na}$ Fase I, quando os fungos dispunham de sacarose, as reduções médias da cor, medidas em função da absorção do grupo cromóforo e em mg Pt.L ${ }^{-1}$, foram, respectivamente de $77 \pm 15 \%$ e $82 \pm 10 \%$, e, na Fase II, quando sua adição no afluente foi interrompida, esses valores foram superiores, registrando-se remoção média do valor da absorção do corante de $85 \pm 10 \%$ e de cor real, de $89 \pm 7 \%$.

Embora os melhores percentuais médios de remoção da cor real tanto em mg Pt. $L^{-1}$ quanto em relação à diminuição do valor da absorção do grupo cromóforo do corante tenham sido alcançados na Fase II, a presença de cossubstrato é relatada como benéfica aos micro-organismos, pois contribui para diminuir a fase lag, período de adaptação ao meio (GRIFFIN, 1994; SINGH, 2006), devendo ser fonte de carbono e de energia de fácil assimilação e de reatividade elevada.

De acordo com Singh (2006), o poluente, menos reativo, tem sua remoção do meio melhorada ao se envolver em reações secundárias com os produtos formados durante a oxidação enzimática do substrato de fácil assimilação.

De qualquer forma, não se pode afirmar que, nesta pesquisa, o sistema empregado tenha trabalhado com eficiência superior no momento da interrupção da adição de sacarose na água residuária devido à curta duração da Fase II, de apenas 21 dias. Faz-se importante operar por mais tempo o reator sem adição de sacarose no afluente, a fim de avaliar seu desempenho sob esta condição operacional.

Ainda que não se tenha quantificado a parcela de corante adsorvida na biomassa - tanto a retida no interior do reator quanto a que porventura tenha deixado o sistema juntamente com pedaços de biomassa presentes no efluente -, nem avaliado possíveis efeitos da ação da luz ambiente sobre a sua oxidação, os resultados do ensaio de adsorção no material suporte revelam que a maior parte do corante afluente foi removida pela ação dos micro-organismos (remoção passiva e assimilação) presentes no reator.
Esta hipótese foi cogitada pelo fato de a manta ter apresentado capacidade para adsorver $0,0001 \mathrm{~g}$ de corante por grama do suporte, de modo que, ao longo dos 75 dias de operação do reator, foi removida uma massa total de corante de $218 \mathrm{mg}$, equivalente a 0,001 g de corante por grama de suporte, valor dez vezes superior à massa de corante necessária para a saturação da manta.

Em relação à matéria orgânica carbonácea, foram alcançadas remoções médias de $71 \pm 16 \%$ e $80 \pm 16 \%$, respectivamente, de matéria orgânica total e dissolvida, na Fase I. A partir do $55^{\circ}$ dia de operação (Fase II), a DQO afluente diminuiu de $518 \mathrm{mg} . \mathrm{L}^{-1}$ para 136 mg. $\mathrm{L}^{-1}$. e a eficiência média de remoção de matéria orgânica foi inferior à registrada na fase anterior, de $44 \pm 47 \%$ de matéria orgânica bruta e $75 \pm 13 \%$ de matéria orgânica dissolvida.

Observou-se também que, para uma mesma amostra do afluente ao reator, ocorreram pequenas diferenças na concentração de matéria orgânica bruta em relação à dissolvida, podendo indicar a presença de micro-organismos no reservatório de armazenamento, ainda que a água residuária sintética tenha sido preparada a cada dois dias para preenchimento do reservatório de alimentação, já que não se objetivou trabalhar com sistema sob condições assépticas.

Particularmente na Fase I, a remoção de cor atingiu valores relativamente próximos aos de matéria orgânica dissolvida na maior parte do experimento, o que pode estar relacionado ao consumo de subprodutos, originados a partir do uso do corante pelos micro-organismos, indicando que, nesta fase, o reator provavelmente produziu efluente com concentração baixa de subprodutos indesejáveis, o que não ocorre em sistemas anaeróbios.

Conforme relatado por Franciscon et al (2009), em sistemas biológicos anaeróbios, a degradação de corantes resulta na formação de subprodutos mais tóxicos que o composto original, os quais têm características mutagênicas e podem ser prejudiciais ao homem, exigindo que o efluente anaeróbio seja submetido a um pós-tratamento em unidade aeróbia.

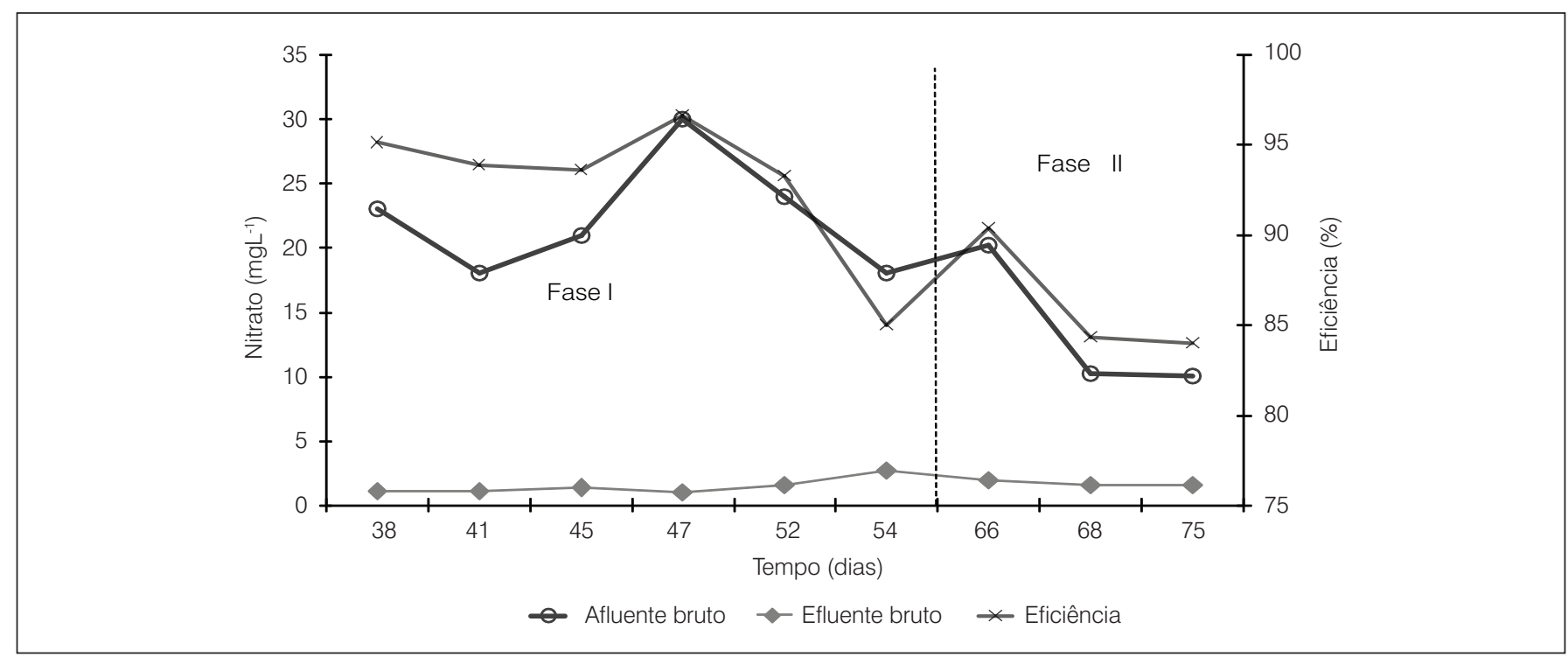

Figura 5 - Variação da concentração de nitrato no afluente e efluente do reator biológico inoculado com fungos 
Somasiri et al (2008), ao estudarem o tratamento de água residuária de indústria têxtil por reator anaeróbio de manta de lodo, sob TDH de 24 horas e com $6.000 \mathrm{mg}$ DQO. $\mathrm{L}^{-1}$ afluente e carga orgânica volumétrica de $0,21 \mathrm{~kg} \cdot \mathrm{L}^{-1} \cdot \mathrm{dia}^{-1}$, obtiveram remoções superiores a 90 e 92\%, respectivamente para cor e matéria orgânica. Porém, segundo os autores, ao longo do experimento, verificouse o acúmulo de subprodutos oriundos da quebra da estrutura do corante, resultando em perda da eficiência de remoção de matéria orgânica.

Nesta pesquisa, foram aplicadas cargas orgânicas volumétricas superiores às de Somasiri et al (2008), de 1,5 kg.L L dia $^{-1}$ (Fase I) e 0,4 kg. $\mathrm{L}^{-1} \cdot \mathrm{dia}^{-1}$ (Fase II). Embora os percentuais alcançados de remoção de matéria orgânica e da cor tenham sido menores em relação aos trabalhos citados, a remoção de matéria orgânica acompanhou a de cor no momento da adição de sacarose no afluente. Deste modo, na Fase I, talvez os metabólitos gerados a partir da utilização do corante, aparentemente, também tenham sido assimilados, o que não ocorreu na pesquisa de Somasiri et al (2008).

Em outro trabalho, Yang et al (2008) submeteram reator aeróbio, inoculado com fungos e bactérias, para tratar meio sintético contendo $30 \mathrm{mg} \cdot \mathrm{L}^{-1}$ de corante preto 5 reativo, a três condições diferentes de alimentação: alta concentração de glicose $\left(5\right.$ g. $\left.\mathrm{L}^{-1}\right)$, concentração intermediária de glicose $\left(1\right.$ g. $\left.\mathrm{L}^{-1}\right)$ e concentração baixa de glicose $\left(0,5\right.$ g. $\left.\mathrm{L}^{-1}\right)$. A melhor eficiência do sistema ocorreu na situação de concentração baixa de glicose, chegando a efluente final com matéria orgânica, medida em DQO, de 21 mg. $\mathrm{L}^{-1}$ a $37 \mathrm{mg} \cdot \mathrm{L}^{-1} \mathrm{e}$ remoções de até $75 \%$ de corante e 94\% de DQO. As menores eficiências foram alcançadas quando da operação com 5 g.L-1 de glicose, tendo-se registrado percentual inferior a 70\% de remoção de DQO, com a predominância de espécies fúngicas em relação às bactérias nas três fases de alimentação estudadas.

Assim como relatado por Yang et al (2008), nesta pesquisa, observou-se que o reator, ao ser alimentado com concentração baixa de cossubstrato, 0,5 g.. $\mathrm{L}^{-1}$ de sacarose, também atingiu excelente percentual de remoção de matéria orgânica de $95 \%$ no $52^{\circ}$ dia.

Foram registrados percentuais maiores que $80 \%$ de eficiência na remoção de nitratotanto na Fase I (93\%) quanto na Fase II (83\%), conforme apresentado na Figura 5.

Segundo Jennings (1995), espécies do gênero Aspergillus têm a capacidade de converter nitrato e nitrito à amônia, forma diretamente assimilada. O nitrato é convertido ao nitrito e este à amônia, mediante a ação das enzimas nitrato reductase e nitrito reductase, respectivamente, as quais são secretadas pelos fungos.

Uma vez que não foi adicionado sal de nitrato ao meio sintético, sua presença no afluente foi atribuída à contaminação da água residuária por bactérias aeróbias, no reservatório de entrada, como resultado do processo de nitrificação. Ressalta-se que o reservatório que armazenava o afluente era periodicamente homogeneizado, procedimento que, consequentemente, introduzia parcela de ar na massa líquida.

Contudo, o nitrato teria sido prontamente utilizado pelos fungos, micro-organismos predominantes no reator, conforme as análises de microscopia, realizadas no final do experimento. Estas análises mostraram ser o Aspergillus niger a espécie predominante e revelaram a existência de leveduras e de outras espécies de fungos filamentosos, bem como de bactérias, e esta microfauna contribuiu para o alcance das eficiências obtidas no processo de tratamento.

A maior remoção de nitrato registrada na Fase I foi atribuída à presença da sacarose, fonte energética prontamente metabolizada, formada por uma molécula de glicose e outra de frutose (MARZZOCO; TORRES, 1999), sendo que a presença deste composto contribui para melhorar a remoção de nitrogênio do meio pelos fungos (PAPAGIANNI, 2007).

Em relação à amônia, sua concentração no efluente final variou de 7,5 mg. $\mathrm{L}^{-1}$ a $13 \mathrm{mg} \cdot \mathrm{L}^{-1}$. A remoção de amônia do meio foi muito baixa, com percentuais máximos de remoção de $25 \%$ ( $10^{\circ}$ dia) na Fase I e de 14\% no último dia de operação (Fase II).

$\mathrm{Na}$ presente pesquisa, particularmente em relação ao nitrato, maiores eficiências foram alcançadas com o uso da sacarose. Contudo, a remoção de amônia não foi eficiente mesmo com a adição de 0,5 g. $\mathrm{L}^{-1}$ de sacarose, o que pode estar relacionado à necessidade de sua disponibilidade em maior concentração no meio para suprir os requisitos metabólicos dos micro-organismos para a produção de aminoácidos.

De acordo com Motta (2005), a glicose que, no caso deste trabalho, estava presente na molécula de sacarose possui papel relevante na assimilação de amônia. Isto ocorre porque a amônia, ao ser armazenada nos vacúolos, no interior da célula fúngica, combina-se com $\propto$-acetoglutarato, composto orgânico intermediário do ciclo tricarboxílico que é gerado a partir da fermentação da glicose (GRIFFIN, 1994; MOTTA, 2005), para formar o aminoácido glutamato, dando início à síntese de aminoácidos.

Porém, o excesso de amônia é liberado para o meio (MOTTA, 2005), o que justificaria os pequenos aumentos de sua concentração no efluente no $12^{\circ}(33 \%), 19^{\circ}(9 \%)$ e $38^{\circ}$ dias (5\%).

A libação de amônia também está relacionada ao processo de homeostase, no qual há liberação ou captação de íons $\mathrm{H}^{+}$e de $\mathrm{NH}_{4}{ }^{+}$ do interior dos vacúolos para o meio onde os micro-organismos se desenvolvem, a fim de manter o $\mathrm{pH}$ intracelular em equilíbrio com o pH do meio externo (LI; KANE, 2008)

Paralelamente, nos dias em que ocorreu aumento da concentração de amônia no meio, o pH do efluente apresentou valores mais elevados em relação aos demais dias, os quais foram de 8,0, 7,0 e 7,10 , respectivamente no $12^{\circ}, 19^{\circ}$ e $38^{\circ}$ dias (Fase I). Na Fase II, o $\mathrm{pH}$ do meio no $66^{\circ}$ e $68^{\circ}$ dias apresentou os maiores valores, de 5 e 6 , quando foram registrados aumentos da concentração de nitrogênio 
amoniacal de 12 e $8 \%$, respectivamente. $\mathrm{O}$ pH da entrada variou de 3,2 a 5,9 (Fase I) e de 4,4 a 6,0 (Fase II).

\section{Conclusões}

A utilização de reatores com Aspergillus niger AN400 pode ser viável para a remoção de cor e de matéria orgânica de águas residuárias contendo corante vermelho do congo, ainda que deva ser levada em consideração a participação dos micro-organismos contaminantes, levedura e bactérias no processo de tratamento.

A adição de sacarose como cossubstrato favoreceu a eficiência na remoção de matéria orgânica. No entanto, a remoção da cor foi aparentemente maior na ausência de sacarose. Registraram-se percentuais de redução da cor em função absorção do grupo cromóforo do vermelho do congo ( $\lambda 500 \mathrm{~nm}$ ) e em mg Pt.L $\mathrm{L}^{-1}$, respectivamente, de $77 \pm 15 \%$ e de $82 \pm 10 \%$ (0,5 g. L $^{-1}$ de sacarose $)$ e de $85 \pm 10 \%$ e de $89 \pm 7 \%$ (sem sacarose); e, em relação à remoção de matéria orgânica dissolvida, de $80 \pm 16 \%\left(0,5\right.$ g.L $\mathrm{L}^{-1}$ de sacarose $)$ e de $75 \pm$ $13 \%$ (sem sacarose).

Quanto à remoção de nutrientes, especificamente de amônia, os resultados mostraram-se insatisfatórios, o que foi atribuído à necessidade de maior concentração de sacarose, cossubstrato utilizado, no afluente. Contudo, um novo experimento, que compreenda um período maior de operação do reator com alimentação sem adição de sacarose deve ser realizado para maior esclarecimento do processo com a ausência de cossubstrato.

\section{Agradecimentos}

Os autores agradecem ao Conselho Nacional de Desenvolvimento Científico e Tecnológico (CNPq) pela concessão das bolsas de iniciação científica.

\section{Referências}

APHA. Standard methods for the examination of water and wastewater. $20^{\text {th }}$ ed. Washington: American Public Association, 1998.

ARAÚJO, A.M. De. Tratamento anaeróbio do líquido do coco verde utilizando reator UASB. 88f. Dissertação (Mestrado em Saneamento Ambiental) - Universidade Federal do Ceará, Fortaleza, 2008.

ARICA, M.Y.; BAYRAMO LU, G. Biosorption of Reactive Red-120 dye from aqueous solution by native and modified fungus biomass preparations of Lentinus sajor-caju. Journal of Hazardous Materials, v. 149, n. 2, p. 499-507, 2007.

CAMPOS, A.C. De; De PAULA, N.M. A indústria têxtil brasileira em um contexto de transformações mundiais. Revista Econômica do Nordeste, v. 37, n. 4, p. 593-608, 2006.

DOS SANTOS, A.B. Pré-tratamento de Águas Residuárias de Indústrias Têxteis Empregando o Processo de Lodos Ativados em Batelada. 120f. Dissertação. (Mestrado de Engenharia Civil, área de concentração em Saneamento Ambiental) - Departamento de Engenharia Hidráulica e Ambiental, Universidade Federal do Ceará, Fortaleza, 2001

FRANCISCON, E. et al. Biodegradation of textile azo dyes by a facultative Staphylococcus arlettae strain VN-11 using a sequential microaerophilic/aerobic process. International Biodeterioration \& Biodegradation, v. 63, n. 3, p. 280-288, 2009.

FU, Y., VIRARAGHAVAN, T. Removal of Congo Red from an aqueous solutions by fungus Aspergillus niger. Advances in Environmental Research, v. 7, n. 1, p. 239-247, 2002.

GRIFFIN, D.H. Fungal physiology. 2 ed. New York: Wiley-Liss, 1994.

GUARATINI, C.C.T., ZANONI, M.V.B. Corantes têxteis. Química Nova, v. 23, n. 1, p. $71-78,2000$.
JENNINGS, D.H. The physiology of fungal nutrition. United Kingdom: Cambridge University Press, 1995.

$\mathrm{KHELIFI}$, E. et al. Aerobic decolourization of the indigo dye-containing textile wastewater using continuous combined bioreactors. Journal of Hazardous Materials, v. 152, n. 2, p. 683-689, 2008.

KUMARE, K.; ABRAHAM, T.E. Biosorption of anionic textile dyes by nonviable biomass of fungi and yeast. Bioresource Technology, v. 98, n. 9, p. 1704-1710, 2007.

LI, S.C.; KANE, P.M. The yeast lysosome-like vacuole: endpoint and crossroads. Biochimica et Biophysica Acta, v. 1793, n. 4, p. $650-$ 663, 2009.

MARZZOCO, A.; TORRES, B.B. Bioquímica básica. Rio de Janeiro: Guanabara Koogan, 1999.

MENDEZ-PAZ, D.; OMIL, F.; LEMA, J.M. Anaerobic treatment of azo dye acid orange 7 under fed-batch and continuous conditions. Water Research, v. 39, n. 5, p. 771-778, 2005

MOTTA, V.T. Bioquímica. Caxias do Sul: EDUCS, 2005.

NACHIYAR, C.V.; RAJAKUMAR, G.S. Mechanism of navitan fast blue s5r degradation by Pseudomonas aeruginosa. Chemosphere, v. 57, n. 3, p. 165-169, 2004.

ONG, S. et al. Simultaneous removal of color, organic compounds and nutrients in azo dye-containing wastewater using up-flow constructed wetland. Journal of Hazardous Materials, v. 20, n. 2, p. 225-228, 2008

PAPPAGIANNI, M. Advances in citric acid fermentation by Aspergillus niger: Biochemical aspects, membrane transport and modeling. Biotechnology Advances, v. 25, n. 3, 244-263, 2007. 
PATEL, R; SURESH, S. Kinetic and equilibrium studies on the biosorption of reactive black 5 dye by Aspergillus foetidus. Bioresource Technology, v. 99, n. 1, 51-58, 2008.

PESSOA-WANDERLEY, C.R. Aspergillus niger AN400 como inóculo de reatores em batelada para remoção do corante vermelho do congo em meio aquoso sintético. 71f. Dissertação (Mestrado em Saneamento Ambiental) - Universidade Federal do Ceará, Fortaleza, Ceará, 2007.

RODRIGUES, K.A. Uso de reator biológico com fungos para remoção de fenol de água residuária sintética com fenol. 144f. Tese. (Doutorado em Hidráulica e Saneamento) - Escola de Engenharia de São Paulo, Universidade de São Paulo, São Carlos, 2006.

SAMPAIO, G.M.M.S. Remoção de metil paration e atrazina em reatores com fungos. Tese (Doutorado em Hidráulica e Saneamento) - Escola de Engenharia de São Carlos, Universidade de São Paulo, São Carlos, 2005.

SANTOS, E.V.M. et al. Avaliação de dois TDH no tratamento biológico de efluente têxtil em reatores com fungos. In: II CONGRESSO DE PESQUISA E INOVAÇÃO TECNOLÓGICA DA REDE NORTE NORDESTE DE EDUCAÇÃO TECNOLÓGICA, Anais... João Pessoa, 2007.

SHARMA, P. et al. Response surface methodological approach for the decolorization of simulated dye effluent using Aspergillus fumigatus fresenius. Journal of Hazardous Materials, v. 161, n. 2, p. 1081-1086, 2009.
SILVA FILHO, H.A. Ensaios de toxicidade e remoção de corantes têxteis por processo biológico. 56f. Trabalho de Conclusão de Curso (Graduação em Tecnologia em Gestão Ambiental) - Instituto Federal de Educação, Ciência e Tecnologia do Ceará, Campus Fortaleza, 2006.

SINGH, H. Mycorremediation. New Jersey: John Wiley \& Sons, 2006.

SOMASIRI, W. et al. Evaluation of the efficacy of up flow anaerobic sludge blanket reactor in removal of colour and reduction of COD in real textile wastewater. Bioressource Technology, v. 99, n. 9, p. 3692 3699, 2008.

D'SOUZA, D.T. et al. Enhanced production of laccase by a marine fungus during treatment of colored effluents and synthetic dyes. Enzyme and Microbial Technology, v. 38, n. 3, p. 504-511, 2006.

SVOBODOVA, K. et al. Mechanism of reactive orange 16 degradation with the white rot fungus Irpex lacteus. Process Biochemistry, v. 42, n. 9, p. 1279-1284, 2007.

YANG, Q. et al. Degradation of synthetic reactive azo dyes and treatment of textile wastewater by a fungi consortium reactor. Biochemical Engineering Journal, v. 43, n. 3, p. 225-230, 2008.

VAN DER ZEE, F. P.; VILLAVERDE, S. Combined anaerobic-aerobic treatment of azo dyes-A short review of bioreactor studies. Water Research, v. 39, n. 8, p. 1425-1440, 2005. 